\title{
Reconceptualizing ER physician wellness in the midst of the pandemic: survival through the lens of personal agency
}

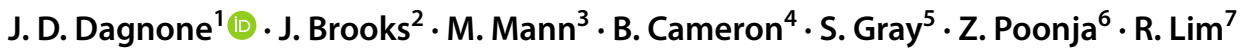

Received: 27 July 2021 / Accepted: 10 December 2021 / Published online: 20 December 2021

(c) The Author(s), under exclusive licence to Canadian Association of Emergency Physicians (CAEP)/ Association Canadienne de Médecine d'Urgence (ACMU) 2021

\section{Introduction}

Emergency medicine physicians are experiencing suffering like no previous time in the history of the profession. Even prior to the COVID-19 pandemic, moral injury as a consequence of long patient waits, barriers in access and hallway medicine was rampant. The state of the Canadian healthcare system has placed an enormous burden on emergency physicians, and despite frontline emergency physicians exhibiting remarkable resilience, the system has created such an unhealthy workplace that almost $85 \%$ suffer from burnout and poor physician mental health as a consequence [1]. During the COVID-19 pandemic, ED staff coined as "health care heroes" have now been subjected to hospital protests and political backlash surrounding compulsory vaccination. Many are suffering from PTSD and are poised to leave their profession at alarming rates [2].

Despite recent attention to many possible strategies advocating for physician wellness, these efforts have not translated into meaningful positive change [3]. The COVID-19 pandemic has amplified this crisis, with physicians feeling

$\triangle$ J. D. Dagnone

damon.dagnone@queensu.ca

1 Emergency Medicine, Queen's University, Kingston, ON, Canada

2 College of Medicine, University of Manitoba, Winnipeg, MB, Canada

3 Emergency Medicine, Stratford General Hospital, Stratford, ON, Canada

4 Michael Garron Hospital, Toronto, ON, Canada

5 St. Michael's Hospital, Toronto, ON, Canada

6 Emergency Medicine, Vancouver General Hospital, Vancouver, BC, Canada

7 Paediatric Emergency Medicine, Western University, London, ON, Canada increasingly overwhelmed in what has become a multi-year global emergency [4].

How can emergency medicine physicians survive in the current climate and overcome barriers to achieving improved health and wellness for themselves despite limitations of the healthcare system? We recognize that meaningful change must occur at all levels of our professional lives-personal, departmental, hospital-wide, regional, professional associations, and governmental. As a result, "achieving" meaningful change can feel overwhelming: exhaustion, fear, and isolation that physicians experience can fuel an increasing perceived lack of control [4]. It is apparent that existing wellness strategies and large scale "systemic" efforts to reduce physician burnout are not working [3].

We advocate that leveraging the concept of personal agency, 'the feeling of being in the driver's seat when it comes to our own actions' [5], can help to reframe our individual circumstances and change what we thought we couldn't. Adapting our behaviour and mindset by granting ourselves permission to take voluntary action can bolster a feeling of greater control and help us weather the current storm.

In the midst of this national healthcare emergency, we propose the most immediate and impactful intervention to withstand the current immediate crisis is to focus on what we can do as individuals to reclaim a sense of personal control and empowerment over the conditions with which we are faced. As psychologist and author Viktor Frankl [6] suggests "when we are no longer able to change a situation, we are challenged to change ourselves." Focusing on individualized changes alone will not immediately alter the bigger system. However, we argue that it is time to go beyond 'in the moment' personal wellness strategies and break down the barriers to wellness within our immediate control.

Knowing that system change is slower, more complex, and involves numerous stakeholders, we propose an approach to optimizing our own personal agency through a process of prioritizing immediate action, identifying 
individual (protective) barriers, time auditing and anchoring these changes by building a more robust and sustainable support system.

\section{Identify the context}

As emergency medicine physicians, we are accustomed to caring for patients in imperfect environments (hallways, corridors, small resuscitation bays etc.), at all times of day or night (including holidays and weekends), with variable support from other services. When there is a crisis at work, we enthusiastically rise to meet the challenge. Our collective ethos is "this is what we trained for" and "this is who we are". Over time, we have developed patterns of behaviour and routines that value patient care above all else. This becomes integrated into our identity as ED physicians, reinforced daily in our clinical environments, and creates barriers to self-care that are both real and perceived. Over time, these patterns begin to shape and shift our beliefs around the power we have to change our circumstances. Recognizing and appreciating overarching barriers-individual, institutional, and system-based (Fig. 1) — that have been created and largely accepted by physicians due to the culture of medicine is an essential step to understanding the problem $[3,7,8]$.

\section{Perform a personal control audit}

"Make every effort to change things you do not like. If you cannot make a change, change the way you have been thinking. You might find a new solution"-Maya Angelou.

Although strategies and practical tips for personal wellness have never been more important during the pandemic [9], it is increasingly important to examine the barriers to real change - transformative change-at the individual level. Once identified, a 'personal control audit' should be undertaken to identify where individual change can be made. Leveraging personal agency practices, ask yourself the following questions:

- What barriers are within my personal control?

- What can be immediately changed, cut out, delegated or modified?

- What commitments are non-essential?

- What personal mental constructs are hindering me from making change?

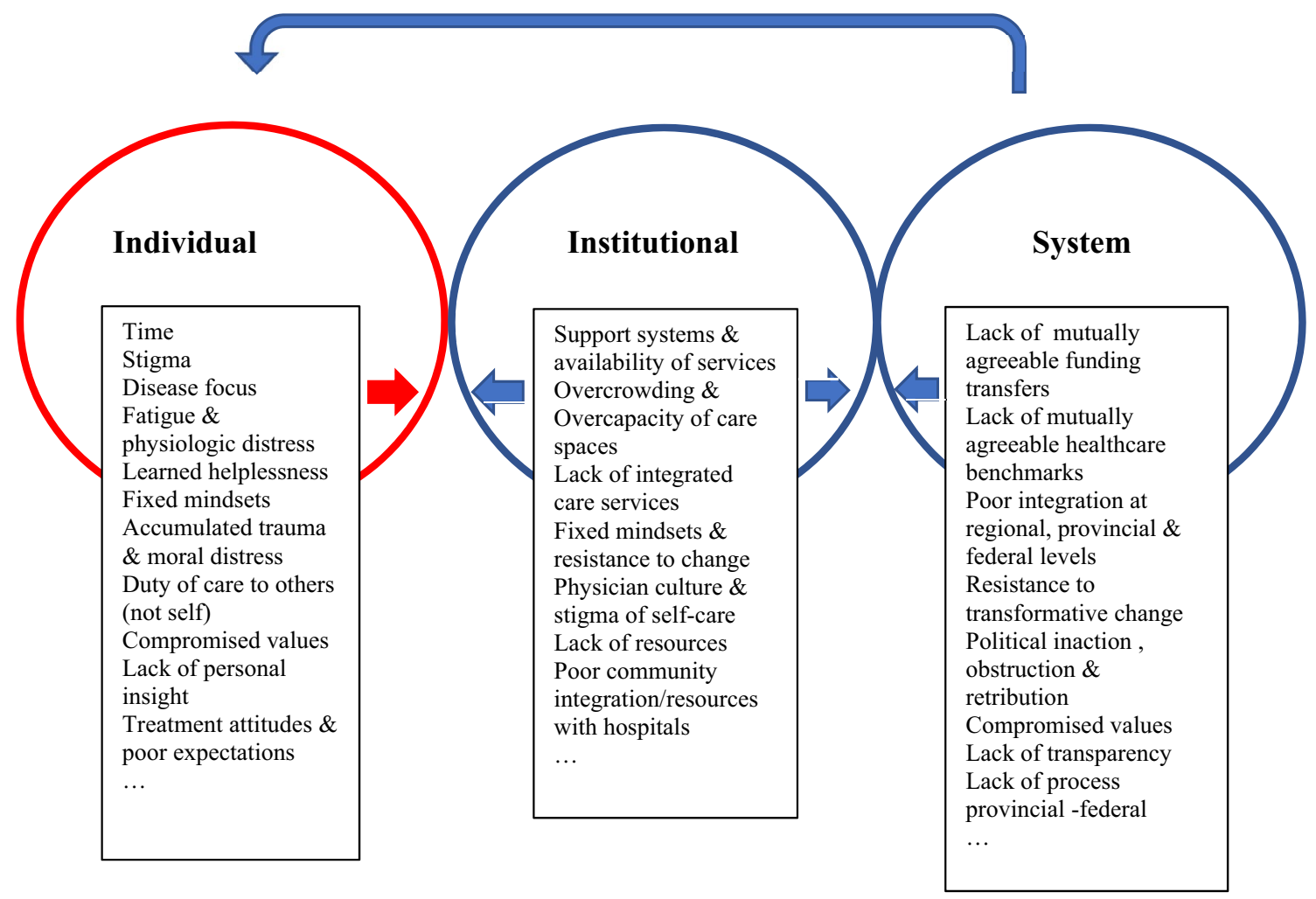

Fig. 1 Individual, institutional \& system barriers to wellness $[3,7,8]$ 
Making change that prioritizes 'self' may be met with resistance-by colleagues, superiors, and family or friends. For example, using the barrier of 'a perceived lack of time', one could decide to work less, enhance childcare help at home, limit social media activity, resign from a committee, or reduce other academic or administrative outputs. In particular, subtracting work to create new time for self is within our control, but may not align with physician culture and expectations, particularly during pandemic times. This will look different for each of us based on our own situations and priorities; however, it is personal agency that is important, more than the look of the plan itself.

\section{Take action}

Once steps for change have been conceptualized, concrete actions are required to embed the new behaviours and beliefs into our core identity. As with committing to any new behaviour, the impact will hinge on how deliberate and consistent one is in executing the actions that support the desired outcome [10]. As an example, many physicians struggle to find time for non-work activities (sports teams, family vacations, book clubs etc.), which may stem from difficulty setting effective boundaries (i.e. saying no). Dedicating time for desired new activities may require the subtraction of other responsibilities such as decreased availability for meetings, canceling or reducing the frequency of lower priority meetings, modifying timelines for career goals, and re-examining the total number of "balls in the air" being juggled at one time. A dedicated approach to creating protected time with a realistic but solid action plan requires deep personal commitment to "me time" and "family time" to counter the powerful pull of physician culture, institutional expectations, and patient care. We need to recognize that often the biggest barrier will be our own perceptions about what we cannot change.

\section{Enhance support networks}

Embarking on a plan to make transformative personal change that prioritizes self requires a community of support. Most emergency physicians require mutual support, so creating shared networks can be beneficial to the group as well as the individual. Sharing changes with colleagues, friends, and family members will help create space for important conversations about ongoing goals, priorities, strengths, weaknesses, vulnerabilities, and personal blind spots. Reaching out to healthcare professionals and accessing available wellness resources (e.g. CAEP, CMA, CMPA, institutional $\&$ provincial wellness programs, therapists, coaches etc.) will help solidify a meaningful future oriented action plan. Building communities for regular non-work related activities (health, outdoors, sports, artistic, spiritual and other endeavours) can also assist with disconnecting from the medical milieu and its blurred boundaries between physician work and life outside healthcare.

\section{Working towards next level change}

Canadian medical training and physician culture causes tension for individual physicians to re-prioritize a focus on 'self' due to the overarching concern for patient welfare first. Instead of conceptualizing patient care vs physician wellness as a binary choice, we need to appreciate that we cannot deliver optimal care when we are personally unwell, overwhelmed, exhausted or burnt out. Improving our personal resilience allows us to be better health care providers.

For many of us, a commitment to improving the healthcare system and emergency medicine care is deeply ingrained in us and can be a source of tremendous pride and satisfaction when improvements are made. Once we acknowledge that institutional and system barriers exist beyond our individual control, our next steps can include combined local and institutional efforts for ongoing advocacy, policy change, and political action [11, 12]. Working with institutional leadership and professional organizations brings opportunities to learn, unlearn, create, and contribute for a greater good. In this fashion, collectively we can work towards bigger goals for institutional and system-level improvements in physician health and wellness.

\section{Conclusion}

The current state of the Canadian healthcare system and the ongoing COVID-19 pandemic puts all emergency physicians at risk of increasing rates of harm, including a myriad of negative mental health outcomes. In these exceptional times, we recommend starting with the prioritization of 'self', as it is important to identify personal change that is feasible, energizing, and immediate as a way of promoting personal agency. Once we prioritize helping ourselves first, we will be better equipped to survive the immense challenges we're facing and then refocus our efforts on institutional and system level changes.

\section{Declarations}

Conflict of interest All authors declare they have no conflicts of interest. 


\section{References}

1. Lim R, Van Aarsen K, Gray S, et al. Emergency medicine physician burnout and wellness in Canada prior to COVID-19: a national survey. CJEM. 2020;22:603-7.

2. CAEP (2021). Emergency department workforce struggles amidst perfect storm. Emergency Department Workforce Struggles Amidst Perfect Storm. https://caep.ca/wp-content/uploads/2021/ 09/ED-Crisis-Sept7.pdf. Retrieved 5 Oct 2021

3. Bynem WE, Varpio L, Tuniessen P. Why impaired wellness may be inevitable in medicine, and why that might not be a bad thing. Med Educ. 2020;55:16-22.

4. Santarone K, McKenney M, Elkbuli A (2020) Preserving mental health and resilience in frontline healthcare workers during COVID-19. Am J Emerg Med 38: 1530-1531. https://www.ajemj ournal.com/article/S0735-6757(20)30258-8/pdf

5. Moore JW. What is the sense of agency and why does it matter? Front Psychol. 2016;7:1272. https://doi.org/10.3389/fpsyg.2016. 01272.

6. Frankl V. Man's search for meaning. London: Rider; 1959.

7. Clough BA, March S, Leane S, Ireland MJ. What prevents doctors from seeking help for stress and burnout? A mixed-methods investigation among metropolitan and regional-based Australian doctors. J Clin Psychol. 2019;75:418-32. https://doi.org/10.1002/ jclp.22707.

8. Naylor CD, Boozary A, Adams O. Canadian federal-provincial/ territorial funding of universal health care: fraught history, uncertain future. CMAJ. 2020;192:E1408-12. https://doi.org/10.1503/ cmaj.200143.

9. Maniuk T, Mok G, Schouela N, Thurgur L, Ho M, Fischer L, Syed S. Beyond survival: practical wellness tips during the 2019 coronavirus disease pandemic. CJEM. 2020;22:579-83. https:// doi.org/10.1017/cem.2020.433.

10. Ericsson A, Pool R. Peak: secrets from the new science of expertise. New York: Houghton Mifflin Harcourt Publishing Co; 2016.

11. Swensen S. Mayo clinic strategies to reduce burnout: 12 actions to create the ideal workplace 12. Oxford Medicine Online 2020; https://doi.org/10.1093/med/9780190848965.001.0001

12. West CP, Dyrbye LN, Erwin PJ, Shanafelt TD. Interventions to prevent and reduce physician burnout: a systematic review and meta-analysis. Lancet. 2016;388(10057):2272-81. 